\title{
Navigation an der Wirbelsäule
}

\author{
Florian Gebhard, Lothar Kinzl, Markus Arand
}

\section{Zusammenfassung}

Computerassistierte und navigationsgesteuerte Operationsverfahren wurden klinisch erstmals im Bereich der Wirbelsäule und bei Neurochirurgischen Eingriffen eingesetzt. Alle Navigationssysteme haben als Grundmodul eine Wirbelsäulenapplikation. Im Bereich der Wirbelsäule stehen prinzipiell zwei Verfahren zur Verfügung: das CT-Daten-basierte Verfahren und das C-Arm-basierte. Die CT-basierte Navigation der Wirbelsäule bietet den Vorteil der präoperativen Planung und weist eine hohe Genauigkeit auf, Letzteres ist insbesondere in anatomisch schwierigen Regionen wie HWS und obere BWS von Vorteil. Die C-Arm-Navigation ist prinzipiell jederzeit intraoperativ im Bereich der ge-

\section{Einleitung}

Die ersten klinischen Anwendungen der computerassistierten Chirurgie (CAS) fanden im Bereich der Orthopädie und Traumatologie an der Wirbelsäule statt (Berlemann et al., 1997; Grützner P.A. et al., 2001; Lavallee et al., 1995; Merloz et al., 1997; Schlenzka et al., 2000b; Schlenzka et al., 2000a; Van Brussel et al., 1996). Die ersten Implantationen von Pedikelschrauben beschränkten sich ausschließlich auf die LWS. Die Weiterentwicklung der Systeme ermöglichte dann auch die Nutzung im Bereich der thorakalen und zervikalen Wirbelsäule.

OP-JOURNAL 2002; 17: 8-14

(c) Georg Thieme Verlag Stuttgart · New York samten Wirbelsäule einsetzbar. Entscheidend hierbei ist jedoch die erreichbare Bildqualität in den einzelnen Anschnitten der Wirbelsäule. Daher ergeben sich oft Einschränkungen der C-Arm basierten Navigation im Bereich der oberen BWS wie auch der HWS. Generell sind beide Verfahren in der Lage, die Präzision der Pedikelschraubenimplantation zu erhöhen. Klinische und experimentelle Studien zeigen eine genaue Pedikelschraubenposition in über $90 \%$ der Fälle. Die CArm-basierte Navigation der Wirbelsäule ist derzeit einer Weiterentwicklung unterworfen: Die Zukunft wird eine intraoperative CT-artige Darstellung der gesamten Wirbelsäule in Kombination mit einer sofort verfügbaren Navigation darstellen.
Darstellungsweise: Je leistungsfähiger der Rechner ist und je höher die Kapazität des Systems, desto besser können Datensätze visualisiert werden.

Für die Navigation selbst ist ein Kamerasystem erforderlich (Abb.1 b). Dieses Kamerasystem dient dazu, die benutzten chirurgischen Instrumente räumlich zu lokalisieren, um deren Position dann an das Rechensystem weiterzugeben.

Es werden im Allgemeinen zwei verschiedene Kamerasysteme benutzt, einmal Systeme die Infrarotlicht empfangen können (Optotrac ${ }^{\circledR}$ ) oder Kamerasysteme die Licht aussenden, das von den Instrumenten reflektiert wird und die reflektierte Strahlung wieder empfangen können (Polaris ${ }^{\circledR}$ ). Physikalisch gesehen können beide Systeme eine annähernd gleiche Ortungsgenauigkeit erreichen.

Experimentelle Daten lassen Systeme mit aktiven LEDs (lichtemittierenden Dioden) an Instrumenten ūnd passiven Kamerasystemen als geringfügig genauer erscheinen.

Klinische Studien zeigen eine Verbesserung der Präzision der transpedikulären Schraubenlage bei Anwendung von CAS-Systemen und gleichzeitig eine Reduktion der intraoperativen Durchleuchtungszeiten (Slomczykowski et al., 1999).

\section{Systeme}

Unabhängig vom gewählten Navigationsmodus (CT oder C-Arm) basieren alle Navigationseinheiten auf so genannten „Workstations“, deren Sinn und Zweck es ist, die zur Operation herangezogenen Bilder in einem sogenannten „real time modus“ in 2D- bzw. 3D-Qualität darzustellen (Abb.1a) (Grützner P.A. et al., 2001; Visarius et al., 1997).

Die Rechengeschwindigkeit dieser Systeme beeinflusst direkt die intraoperative
Im Großen und Ganzen ist das Prinzip der intraoperativen Navigation den Navigationssystemen eines Kraftfahrzeuges vergleichbar. Durch die Interaktion von Kamera (Satellit) und chirurgischem Instrument (Kraftfahrzeug) ist eine geometrische Positionsbestimmung des Instrumentes (Autos) möglich. Die übermittelten Daten werden an das Rechensystem weitergeleitet (Workstation), das dann die räumliche Lage des Instruments (Auto) in Bezug setzt zur Knochenanatomie des Patienten (Landkarte). Klinische Anwendung:

Im Bereich der Wirbelsäule steht die Navigation prinzipiell für alle Wirbelsäulenabschitte zur Verfügung.

In Abhängigkeit von der Lokalisation an der Wirbelsäule bietet sich das CT-basierte (HWS, BWS) oder das C-Arm-basierte (BWS, LWS) Verfahren an. 

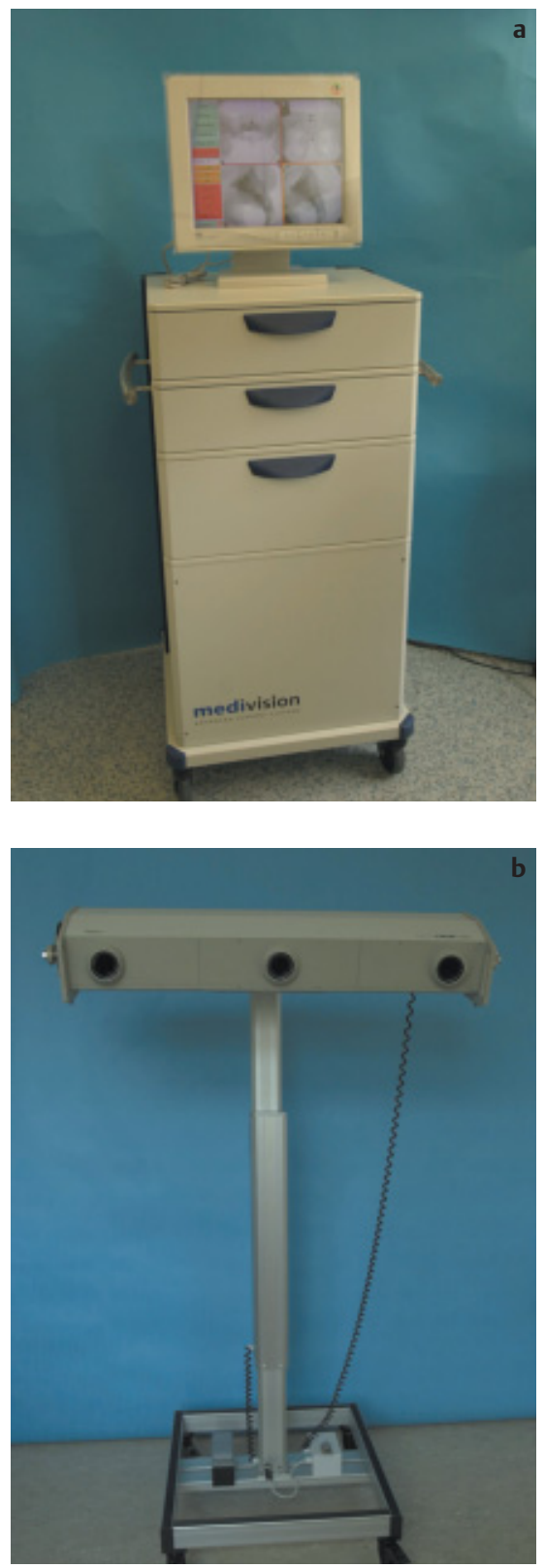

Abb. 1 a u. b Rechnersystem mit Monitor (Surgigate ${ }^{\circledR}$ ) und Kamera (Optotrac ${ }^{\circledR}$ ).

\section{- CT-basierte Navigationen:}

Das CT-basierte Modul beruht auf dem präoperativen CT-Datensatz.

Dieser Datensatz muss den frakturierten Wirbelkörper enthalten sowie einen Wirbel kranial und kaudal des oder der frakturierten Wirbelkörper.

In der Regel kann dabei anhand von 100 bis 150 CT-Schichten eine Lokalisation und Planung der Pedikelinstrumentation erfolgen. Die klinische Erfahrung zeigt, dass es im Bereich der BWS erforderlich ist mehr Wirbelkörper im präoperativen Datensatz zu erfassen, als nur den verletzten Wirbelkörper und die angrenzenden, um eine exakte Höhenlokalisation bzw. Planung durchzuführen. Thorakal müssen prinzipiell die beiden nächst kaudalen Wirbelkörper miterfasst werden, da die Überlappung der thorakalen Dornfortsätze nach kaudal wesentlich ausgeprägter ist als lumbal oder zervikal. Die intakten Dornfortsätze sind wiederum für den so genannten Matching. (Referenzierungs-)Prozess erforderlich.

Es werden daher im Bereich der BWS in der Regel 5 bis 6 Wirbelkörper CT-mäßig gefasst, wobei Schichtzahlen zwischen 200 und 250 Schichten erreicht werden.

Dies ist insofern von Bedeutung, als Navigationssysteme in der Regel eine limitierte Anzahl von rechnerisch verfügbaren Schichten bearbeiten können. In dem bei uns verwendeten System (Surgigate $^{\circledR}$ ) können maximal 250 Schichten bearbeitet werden.

D.h., bei dem systemabhängig vorgeschriebenen Datenerfassungsprotokoll, muss bei der vorgegebenen maximalen Anzahl von 250 Schichten durch entsprechend gewählte Schichtabstände die Scanstrecke eingegrenzt werden. Gleichzeitig ist es wichtig, das Scanfeld des CT auf die Wirbelsäule einzugrenzen (Zoomen).

Für die CT-basierte Navigation muss dann dieser Datensatz in das Navigationssystem transferiert werden. Dies kann entweder durch direkte Netzanbindung des Navigationsrechners erfolgen oder durch Zwischenspeichern auf einem Transfermedium (EOD, JAZ-Disk).

Anschließend wird dieser Rohdatensatz im Navigationsrechner geladen (Abb.2) und muss dann für die präoperative Planung aufbereitet werden. Im Einzelnen muss hierbei der Schwellenwert für Grauwerte festgelegt werden (Threshold). Dies geschieht manuell. Ziel ist es, dass die Knochenoberfläche vom Rechensystem möglichst präzise erkannt wird, da dies die Grundlage aller weiteren Abschnitte der Navigation ist (Abb.3).

Bei der Planung der Knochenoberfläche ist diese so vorzunehmen, dass Artefakte unterdrückt werden bzw. nicht zu einer falschen Knochenoberflächendarstellung führen. Schwierig ist dieser Vorgang bei osteoporotischem Knochen, da hier, insbesondere thorakal, die Knochendichte
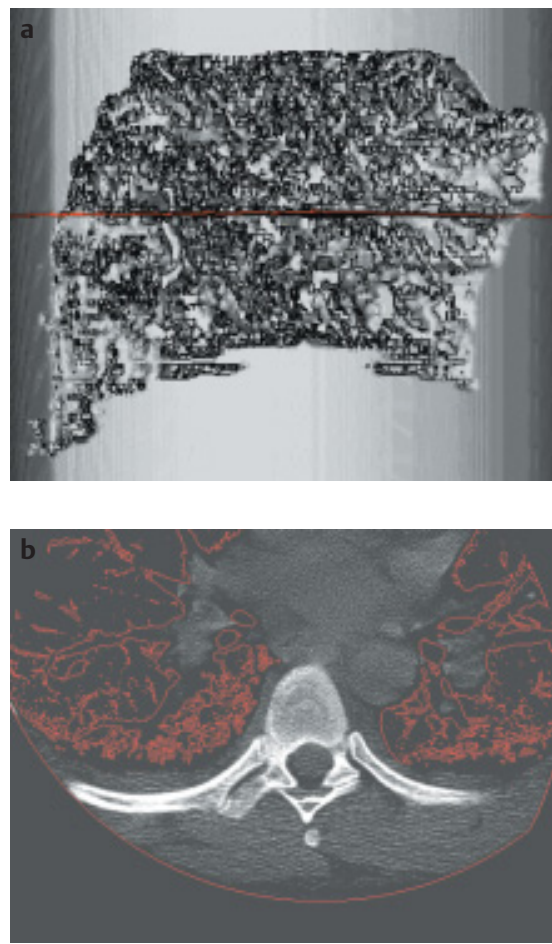

Abb.2au.b Rohdatensatz einer thorakalen Wirbelsäule, der Rechner bildet das Lungengewebe (rot umrandet) ab.
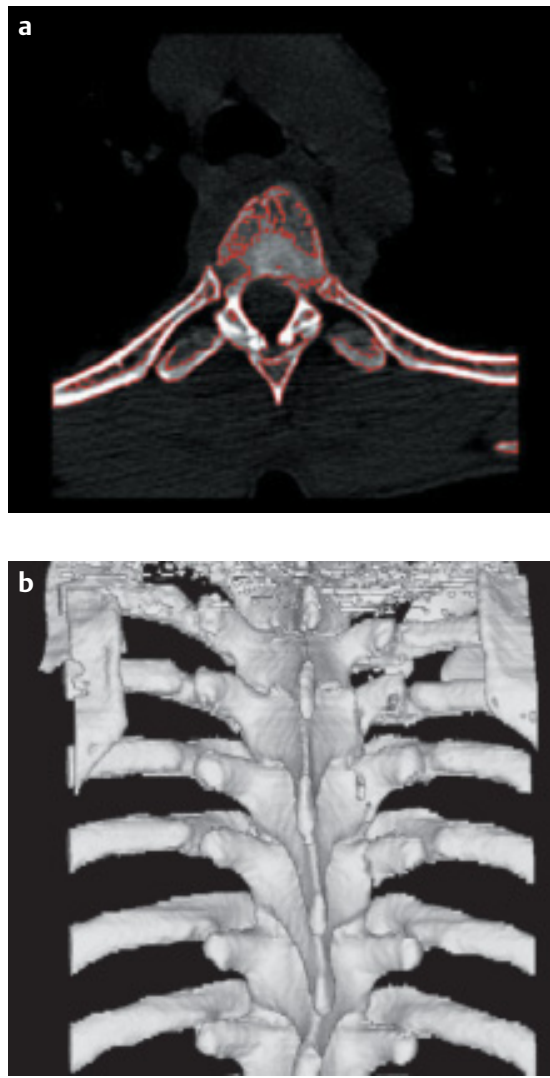

Abb.3au.b Festlegen der Knochengrenzen durch Schwellenwerte. Der Rechner zeigt nun die Knochenoberfläche (rot umrandet). 
der Rippen die der Wirbelkörper bei Weitem übersteigt.

Eine nicht korrekt errechnete Oberfläche im Rahmen eines Planungsvorgangs führt zu gefährlichen Fehlern während der klinischen Anwendung.

Weitere Fehlermöglichkeiten zu diesem Zeitpunkt bestehen darin, dass nicht alle CT-Schichten übertragen werden. Fehlende Schichten führen zu Fehlern in der Rekonstruktion der Wirbelkörperhöhe.

\section{Planung:}

Nach erfolgreicher Übertragung aller Schichten und Definition der Knochenoberfläche kann die Planung erfolgen.

Um beim CT-basierten Verfahren dem Navigationssystem mitzuteilen, in welcher Position sich der Knochen im OPSaal befindet, muss ein sogenannter Matchingvorgang durchgeführt werden.

D.h. hier wird intraoperativ der Abgleich zwischen der virtuellen Welt des CT-Datensatzes und der realen Welt des Patienten auf dem Operationstisch durchgeführt. Da davon auszugehen ist, dass die Lage des Patienten auf dem Operationstisch nicht der Lage der Wirbelkörper im CT entspricht, hat diese so genannte Matchingwirbelkörperweise zu erfolgen.

Dies geschieht, indem in der Planungsphase wieder-auffindbare Punkte definiert werden. Bei der Definition der Punkte für das Paired-point-Matching ist zu berücksichtigen, dass sie so gewählt werden, dass sie mit einer hohen Wahrscheinlichkeit intraoperativ auch wiedergefunden werden können. Darüber hinaus ist es wichtig darauf zu achten, dass die drei bis fünf Punkte möglichst eine dreidimensionale geometrische Fläche aufspannen und nicht in einer Linie oder einer Ebene liegen, da dies die mathematische Zuordnung der Position verbessert bzw. verschlechtert.

Geplant werden können prinzipiell nur Wirbelkörper die nicht frakturiert sind und einen unverletzten Dornfortsatz haben.

Eine Planung eines frakturierten Wirbelkörpers ist problematisch, da dessen Position sich vermutlich durch das Umlagern ändern wird und diese Änderung nicht erfasst werden kann.

Wenn die so genannten Paired-point Punkte definiert wurden, in der Regel zwischen 4 und 5 Punkten, kann anschließend in dem Wirbelkörper die geplante Position der Pedikelschraube eingezeichnet werden als sogenannte Trajektorie (Abb.4).

Wenn sämtliche zu navigierenden Wirbelkörper mit sogenannten Referenzierungspunkten für das Registrierungs-/ Matchingverfahren versehen worden sind und die gewünschte Pedikelschraubenlage eingezeichnet ist, ist der Schritt der präoperativen Planung abgeschlossen.
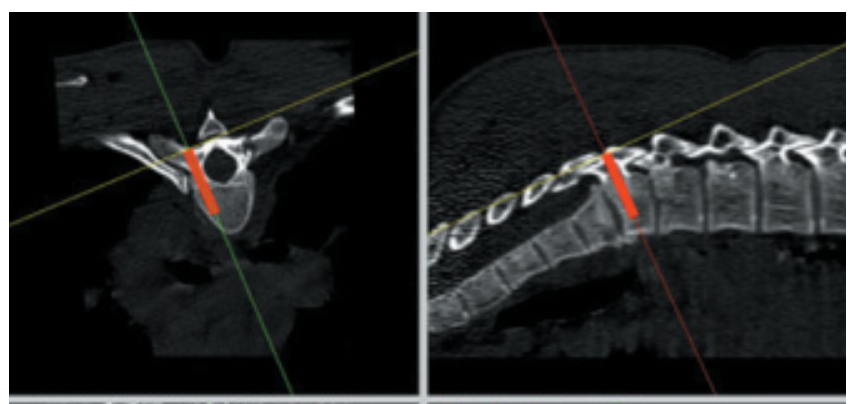

Abb. 4 Geplante Pedikelschraubenlage (Trajektorie) in einem Brustwirbelkörper.

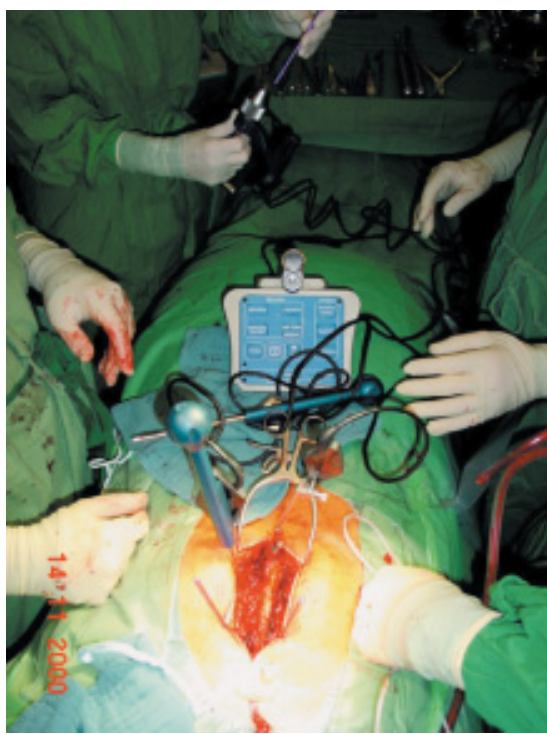

Abb.5 Situs einer Brustwirbelsäule, Navigation des kaudalen Wirbelkörpers. Der kraniale Wirbelkörper (im Bild unten) ist bereits instrumentiert.

\section{Matching}

Intraoperativ erfolgt zunächst die chirurgische Exposition des zu operierenden Wirbelsäulenabschnittes in gewohnter Weise. Die Praxis hat gezeigt, dass für die Navigation, insbesondere thorakal der Zugangsweg nach kaudal etwas weiter als üblich erfolgen muss (Abb.5).

Es wird dann zunächst der erste zu navigierende Wirbelkörper identifiziert. Hierbei ist auch bei der CT-Navigation in der Regel der Bildverstärker heranzuziehen, da das Navigationssystem per se nicht entscheiden kann, welcher Wirbelkörper vorliegt. Anschließend wird dieser identifizierte Wirbelkörper mit einer Markierung versehen (Data reference base: DRB). Diese dient dazu, dem Navigationssystem zunächst mitzuteilen, in welcher Region die Punkte abgegriffen werden und gleichzeitig wird über diese Referenzierungsbasis jegliche weitere Bewegung des Wirbelkörpers ab dem Zeitpunkt des Matchings nachgerechnet, so dass, wenn ein Wirbelkörper einmal erfasst wurde, das System auch Bewegungen desselben verfolgen kann.

Es erfolgt dann das Abgreifen der präoperativ geplanten Punkte (Abb.6), hieraus errechnet das System einen Genauigkeitswert der in etwa angibt, wie präzise diese Punkte in Bezug auf den CT-Datensatz gematcht werden konnten.

Dieser Vorgang muss u.U. mehrfach wiederholt werden, bis eine ausreichende 

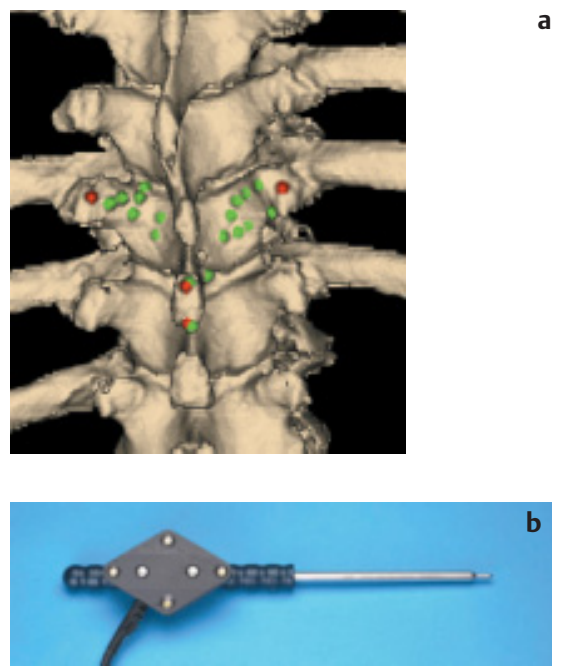

Abb.6au.b Abgeschlossenes Matching: paired point - rote Planungspunkte und surface matching - grüne Punktwolke. Erfasst werden die Punkte mittels eines navigierten Zeigeinstrumentes (pointer).

Genauigkeit erzielt wurde. Die Präzision dieses Matching-Verfahrens kann erhöht werden, indem weitere 12 oder mehr Punkte auf der Oberfläche des Wirbelkörpers abgegriffen werden (Surface-Matching, Abb. 6). Diese willkürlich intraoperativ abgegriffenen Punkte bilden eine mathematische Punktwolke, die mit dem präoperativen Datensatz in Übereinstimmung gebracht werden kann.

Hierbei ist zu beachten, dass das Surface Matching, im Gegensatz zu dem Pairedpoint Matching, auf dem errechneten dreidimensionalen Modell beruht. D.h. eine unpräzise errechnete dreidimensionale Oberfläche (Schwellenwert in der Planungsphase) macht in diesen Fällen das Navigieren unmöglich oder gefährlich, da dem Navigationssystem eine falsche Oberfläche zugrundeliegt. Dadurch kann es zur systemischen Verschiebung sämtlicher Bezugstrukturen mit entsprechender Implantatfehllage kommen, ohne dass das Navigationssystem eine Möglichkeit hat dies zu erkennen.

Um diesen Fehlermöglichkeiten vorzubeugen, ist allen Navigationssystemen ein sogenannter Überprüfungsmodus eingebaut. Dieser Modus (Verification) ermöglicht es mit einem navigierten Zeiger beliebige Punkte auf der Knochenoberfläche des Patienten abzutasten. Das Navigationssystem muss dann in diesen Fällen die gewählte Position exakt wiedergeben. Sollte hier beim Navigationssystem die Instrumentenspitze in den Knochen projiziert werden oder deutlich außerhalb des rekonstruierten CT-Datensatzes liegen, kann davon ausgegangen werden, dass das Navigationssystem nicht die wahre Position des Wirbelkörpers erfasst hat. In diesen Fällen ist dann der sogenannte Matchingprozess zu wiederholen (Gebhard et al., 2000).

\section{Zielführung:}

Sobald der Registrierungsprozess für einen Wirbelkörper abgeschlossen ist, beginnt die eigentliche Implantation der Pedikelschrauben. Hierbei kann unterschieden werden zwischen einem so genannten Zielführungsprogramm (Guidance) oder einer Echtzeitdarstellung (Realtime).

Wie bereits in der Planung ausgeführt, kann man die Pedikelschraubenlage in den CT-Datensatz einzeichnen. In diesen Fällen würde man über den Guidancemodus den Eintrittspunkt der Pedikelschraube durch das System dargestellt bekommen (Abb. 7).

D. h. mit dem Instrument, welches zur Eröffnung des Pedikeleintrittes dient, wird intraoperativ der Punkt aufgesucht der präoperativ als Eintrittspunkt definiert wurde. Anschließend kann dann der Pedikelschraubenkanal mit einem weiteren digitalisierten Instrument ausgetastet werden und letztendlich über einen navigierbaren T-Handgriff die Pedikelschraube eingeführt werden. Nach Einbringen
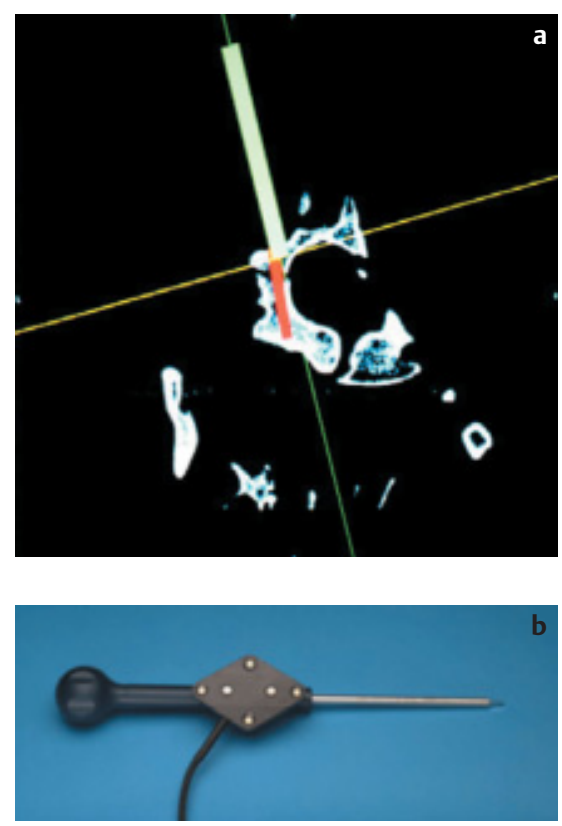

Abb. 7 a u. b Zielführung an einem Halswirbelkörper (Guidance). Eröffnung der Eintrittsstelle mit einem navigierten Pfriem.

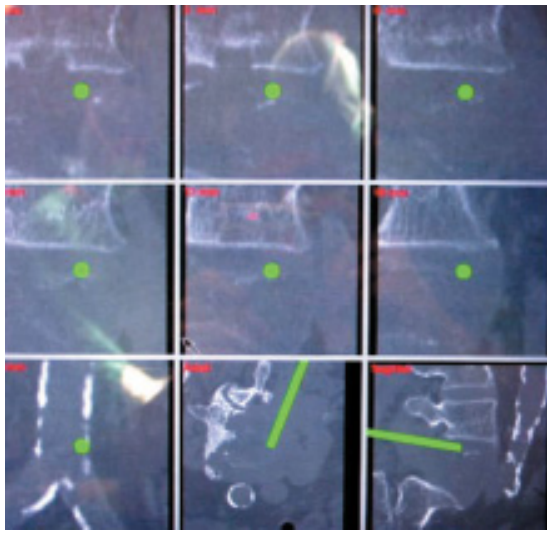

Abb. 8 Realtimedarstellung einer Biopsie bei einem lumbalen Wirbeltumor. Instrumentenposition in Grün.

der beiden Pedikelschrauben in den Wirbelkörper ist dieser Vorgang abgeschlossen. Zur Instrumentierung weiterer Wirbelkörper müssen die Schritte des Matching/Registrierens wieder ausgeführt werden.

Der sogenannte Echtzeitmodus visualisiert die Position eines beliebigen Instrumentes, welches von der Kamera erfasst wird in Bezug auf den CT-Datensatz. Dabei wird bei der Wirbelsäulennavigation eine koronare Schnittführung rekonstruiert und der virtuelle Verlauf des Instrumentes im Datensatz visualisiert. Auf diese Weise kann intraoperativ unabhängig von einer präoperativen Planung eine Pedikelschraubenimplantation in den jeweiligen gematchten Wirbelkörper erfolgen.

Zusätzlich kann dieser Modus auch genutzt werden, um beispielsweise navigierte Biopsien durchzuführen (Abb. 8). Hierbei wird dann das Biopsieinstrumentar visualisiert.

\section{C-Arm-Navigation}

Die C-Arm-basierte Navigation beruht auf den intraoperativ angefertigten Durchleuchtungsbildern mittels eines navigierten C-Arms.

Das Prinzip hierbei ist, dass der intraoperativ verwendete $\mathrm{C}$-Arm mit Elektroden ausgestattet ist, welche von dem Navigationssystem erkannt werden können (Abb.9) (Arand et al., 2001b; Grützner P.A. et al., 2001). Da dem Navigationssystem durch einen einmal durchzuführenden Kalibrierungsvorgang die geometri- 

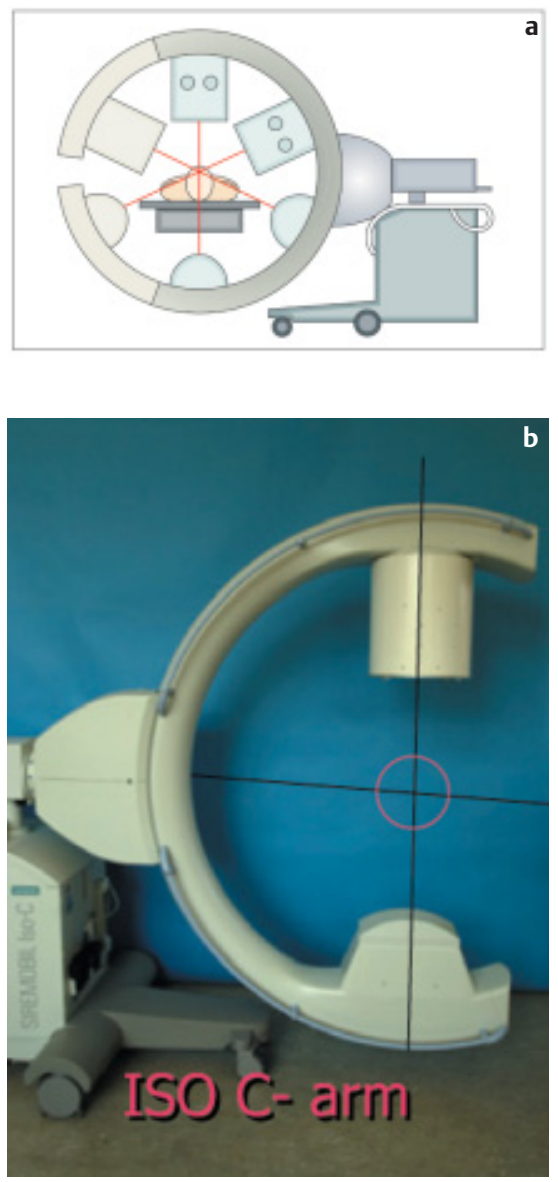

Abb. 9 a u. b Der mit LED ausgestattete C-Arm wird vom Navigationssystem erfasst.

schen Daten des C-Arms sowie dessen Bildmittelpunkt bekannt sind, ist es mathematisch möglich nach Durchführung eines intraoperativen Durchleuchtungsbildes mit dem navigierten C-Arm die Position des Bildes im Raum zu berechnen.

Das bei uns verwendete System kann maximal 4 Röntgenbilder, welche mit dem navigierten C-Arm aufgenommen werden, gleichzeitig darstellen.

Anschließend kann nach Erfassung dieser Bilder jedes navigierte Instrument sofort in den Röntgenbildern visualisiert werden. Ein zusätzliches Matching-Verfahren wie oben beschrieben, ist hierbei nicht erforderlich.

Einschränkend ist für die C-Arm-Navigation zu sagen, dass die zunächst gewonnenen originären zweidimensionalen BV-Bilder aufgrund der physikalischen Struktur der Bildverstärker Verzerrungen im Randbereich aufweisen, so dass eine
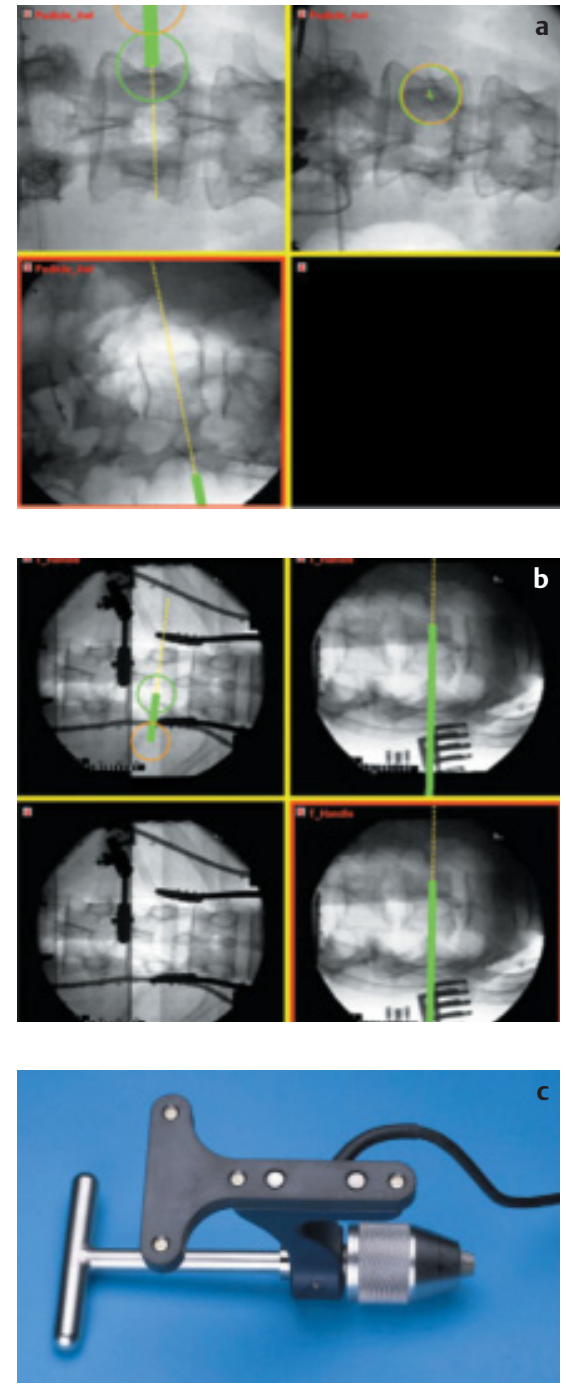

Abb.10a-c Simultane Darstellung aller gewählten Projektionen während der gesamten Instrumentierung. Eindrehen der Pedikelschanzschraube mit dem navigierten T-Handgriff.

entsprechende Korrektur (Entzerrung) erforderlich ist. Diese Korrektur der Verzerrung des Bildverstärkers erfolgt in dem von uns verwendeten System über ein Referenzgitter, das fest im Strahlengang montiert wird. Die hierbei während der Bilderfassung miterfassten Schattenbilder der Kugelstrukturen dienen zur mathematischen Rückrechnung und Entzerrung des Röntgenbildes.

Da aber die C-Arm-Elektronik eine Belichtungsautomatik aufweist, führt das Einbringen dieser röntgendichten Kugelstrukturen zu einer Beeinflussung der Belichtungsautomatik, die in einer Reduktion der Bildqualität mündet.
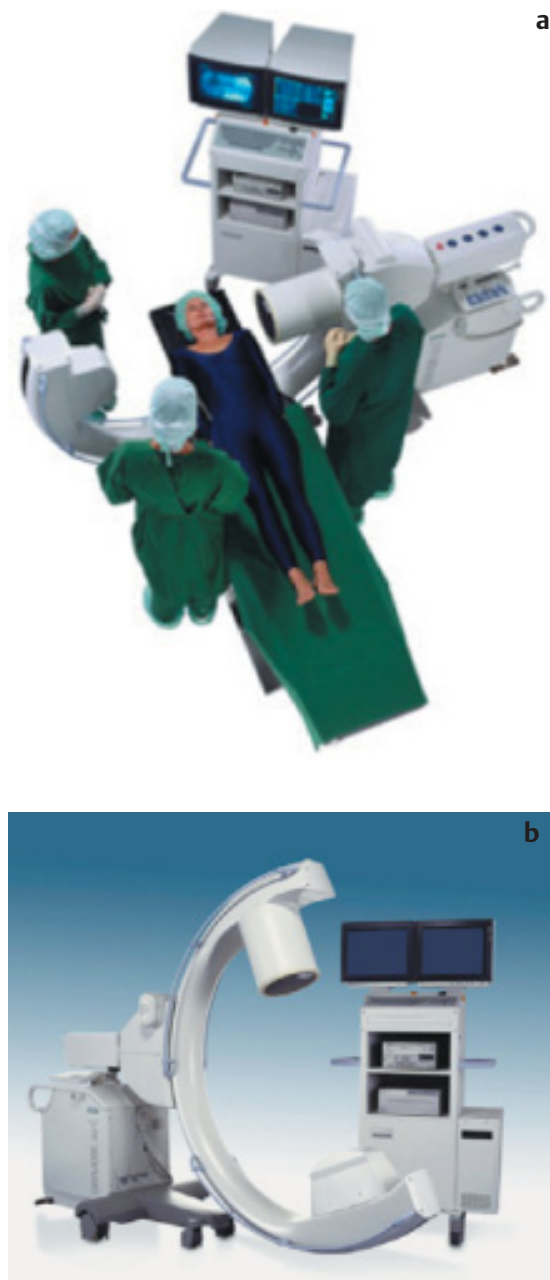

Abb.11a u.b 3D-C-Arm-System.

Ein weiterer Nachteil ist bei der C-ArmNavigation, dass im Bereich der Wirbelsäule bestimmte anatomische Lokalisationen wie die kraniale BWS, die kraniale HWS mit dem Bildverstärker nicht immer in beiden Ebenen ausreichend erfasst werden können. In diesen Fällen ist eine C-Arm-Navigation nicht möglich, da deren Präzision abhängig ist von der Bildqualität.

Vorteil der C-Arm-Navigation ist, dass zunächst intraoperativ beliebige Bilder gemacht werden können (Abb.10) und geeignete Positionen sekundär aus dieser Bildgalerie ausgewählt werden können. Gleichzeitig ist dieser Modus unbeeinflusst von intraoperativen Lagerungsänderungen, d.h. es wird immer das aktuelle Bild dargestellt, so dass durchgeführte Repositionen zu keiner Einschränkung der Navigation führen können, wie dies im CT-basierten Bereich der Fall ist. 

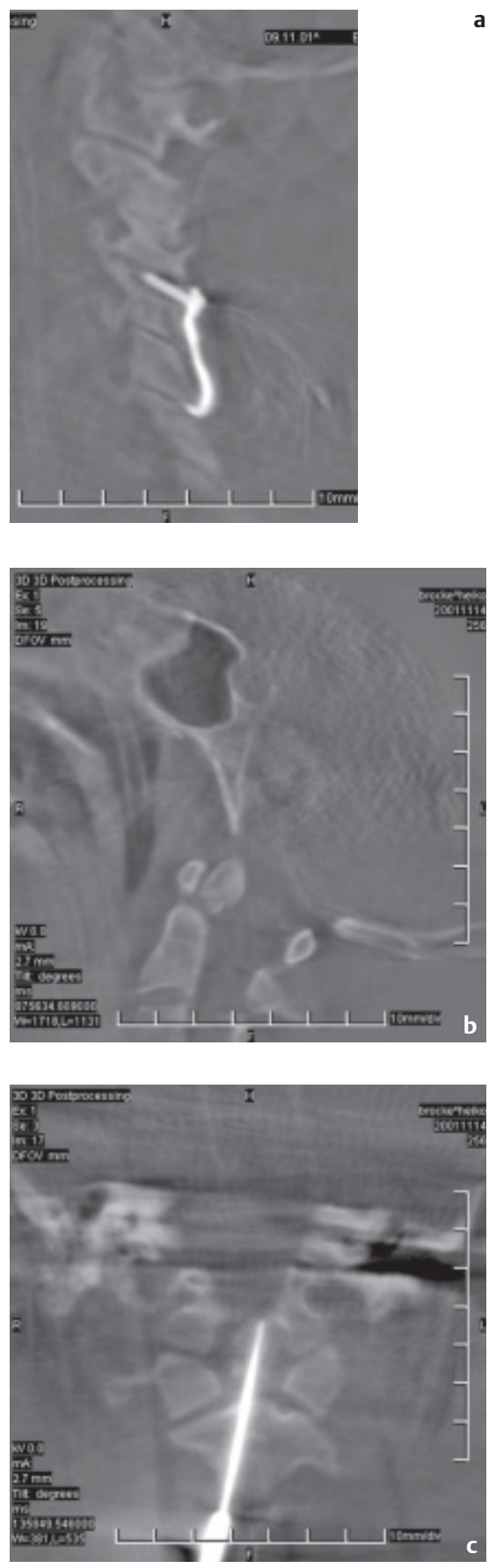

Abb.12a-c Darstellung der Halswirbelsäule im 3D-C-Arm in CT ähnlicher Qualität.

\section{Zukunft}

Seit August 2001 steht ein C-Arm-Modell zur intraoperativen Generierung CT-ähnlicher Daten von Knochen (High density objects) zur Verfügung (Abb.11). Mit diesem System ist es erstmals möglich, CTähnliche Bilder intraoperativ beliebig oft zu generieren.

Da diese Bilder von einem C-Arm erstellt werden, der prinzipiell geeignet ist für die Navigation eingesetzt zu werden, ergibt sich hieraus die Möglichkeit beides zu kombinieren:

Die Weiterentwicklung ist also die intraoperative Erstellung CT-ähnlicher Datensätze, die dann gleich ohne weitere Registrierungsvorgänge zur Navigation genützt werden können.

Auf diese Weise sind die bisherigen Nachteile der beiden Vorgehensweisen an der Wirbelsäule - CT oder C-Arm - beseitigt. Diese neue Applikationsform hat die Vorteile der CT-ähnlichen Visualisierung der Wirbelsäule ohne den Nachteil der so genannten „eingefrorenen präoperativen Realität“, da in diesen Fällen die intraoperative Realität beliebt oft reproduziert werden kann.

Darüber hinaus hat diese neue Modalität im Vergleich zur konventionellen C-ArmNavigation den Vorteil, dass sie aufgrund der dreidimensionalen Bildgebung weitgehend unabhängig ist von Einschränkungen der Bildqualität, wie sie im Bereich der thorakalen Wirbelsäule oder der kranialen HWS bei Standardprojektionen auftreten können (Abb.12).

\section{Einsatzmöglichkeiten}

Zum gegenwärtigen Zeitpunkt kann die computerassistierte Chirurgie im Bereich der Wirbelsäule für alle Wirbelsäulenabschnitte zur dorsalen Instrumentation angewandt werden.

Aus der klinischen Erfahrung heraus empfiehlt sich die CT-basierte Navigation an der Wirbelsäule für

- HWS

- kraniale BWS

die C-Arm-basierte Navigation für

- thorakolumbalen Übergang

- LWS.

einzusetzen.

Für die Akutversorgung der Wirbelsäule bietet die C-Arm-Navigation den Vorteil der universellen und unabhängigen Verfügbarkeit im OP-Bereich, wohingegen die CT-basierte Navigation aufgrund ihrer aufwändigen Datenerhebung und präoperativen Planung eine Einschränkung für den Notfallbereich erfährt.

Komplexe Verletzungen der Wirbelsäule, schwierige Anatomie wie bei Skoliose oder M. Bechterew haben durch die computerunterstützte Navigation im Bereich der Wirbelsäule eine deutliche Verbesserung der Implantatlagen erfahren.
Der universelle Einsatz der computerassistierten Chirurgie im Bereich der Wirbelsäule, auch im Akuttraumabereich, wird mit Einführung der navigierten dreidimensionalen intraoperativen CArm-basierten Navigation zu einem unverzichtbaren Hilfsmittel in der Traumatologie/Orthopädie führen.

\section{Literatur}

${ }^{1}$ Arand M, Hartwig E, Hebold D, Kinzl L, Gebhard F. Präzisionsanalyse navigationsgestützt implantierter thorakaler und lumbaler Pedikelschrauben. Eine prospektive klinische Studie. Unfallchirurg 2001; 104 $1076-1081$

${ }^{2}$ Arand M, Hassfeld S, Kinzl L, Gebhard F. Spinal navigation in cervical fractures - a preliminary clinical study on Judet-osteosynthesis of the axis. Comp Aid Surg 2001; 6: $170-175$

${ }^{3}$ Arand M, Schempf M, Kinzl L, Fleiter T, Pless D, Gebhard F. Präzision standardisierter IsoC-Arm basierter navigierter Bohrungen an proximaler Femur. Unfallchirurg 2001; 104: $1150-1156$

${ }^{4}$ Berlemann U, Langlotz F, Langlotz U, Nolte LP. Computerassistierte Orthopädische Chirurgie (CAOS). Von der Pedikelschraubeninsertion zu weiteren Applikationen. [Computer-assisted orthopedic surgery. From pedicle screw insertion to further applications]. Orthopäde 1997; 26: 463-469

${ }^{5}$ Gebhard F, Arand M, Fleiter T, Hebecker A, Heeckt P, Hesser J, Messmer P, Hufner T, Visarius H, Regazzoni P, Kinzl L. Computer assistierte Chirurgie, Entwicklung und Perspektiven 2001. Ergebnisse einer Arbeitstagung auf Schloss Reisenburg, 23./ 24.11.2000. [Computer-assisted surgery: developments and prospects in 2001. Results of a workshop at Schloss Reisenburg. 23-24 November 2000]. Unfallchirurg 2001; 104: $782-788$

${ }^{6}$ Gebhard F, Kinzl L, Arand M. Computerassistierte Chirurgie. [Computer-assisted surgery]. Unfallchirurg 2000; 103: 612-617

${ }^{7}$ Grützner PA, Vock B, Koehler T, Wentzensen A. Rechnergestütztes Operieren an der Wirbelsäule. OP-Journal 2001; 17: 185-190

${ }^{8}$ Kim KD, Patrick-Johnson J, Bloch-BS O, Masciopinto JE. Computer-assisted thoracic pedicle screw placement: an in vitro feasibility study. Spine 2001; 26: 360-364

${ }^{9}$ Lavallee S, Sautot P, Troccaz J, Cinquin P, Merloz P. Computer-assisted spine surgery: a technique for accurate transpedicular screw fixation using CT data and a 3-D optical localizer. J Image Guid Surg 1995; 1: 65-73

${ }^{10}$ Merloz P, Tonetti J, Cinquin P, Lavallee S, Troccaz J, Pittet L. Chirurgie assistee par ordinateur: vissage automatise des pedicules vertebraux. [Computer-assisted surgery: automated screw placement in the vertebral pedicle]. Chirurgie 1998; 123: 482-490

${ }^{11}$ Merloz P, Tonetti J, Eid A, Faure C, Lavallee S, Troccaz J, Sautot P, Hamadeh A, Cinquin P. Computer assisted spine surgery. Clin Orthop 1997; 86-96

12 Nolte LP, Zamorano L, Arm E, Visarius H, Jiang Z, Berlerman U, Schwarzenbach O. Imageguided computer-assisted spine surgery: a pilot study on pedicle screw fixation. Stereotact Funct Neurosurg 1996; 66: 108 - 117 
${ }^{13}$ Schlenzka D, Laine T, Lund T. Computerassisted spine surgery. Eur Spine J 2000a 9 Suppl 1S57-64:-64

${ }^{14}$ Schlenzka D, Laine T, Lund T. Computerunterstützte Wirbelsaülenchirurgie. Prinzipien, Technik, Ergebnisse und Perspektiven. [Computer-assisted spine surgery: principles, technique, results and perspectives]. Orthopäde 2000b; 29: 658-669

${ }^{15}$ Slomczykowski I, Roberto M, Schneeberger P, Ozdoba C, Vock P. Radiation dose for pedicle screw insertion. Fluoroscopic method versus computer-assisted surgery. Spine 1999; 24: $975-982$
${ }^{16}$ Van Brussel K, Vander-Sloten J, Van Audekercke R, Fabry G. Internal fixation of the spine in traumatic and scoliotic cases. The potential of pedicle screws. Technol Health Care 1996; 4: 365-384

${ }^{17}$ Visarius H, Gong J, Scheer C, Haralamb S, Nolte LP. Man-machine interfaces in computer assisted surgery. Comput Aided Surg 1997; 2: $102-107$
Priv.-Doz. Dr. med. Florian Gebhard Leitender Oberarzt

Prof. Dr. med. Lothar Kinzl

Ärztlicher Direktor

Dr. med. Markus Arand

Oberarzt

Universitätsklinikum Ulm

Abteilung Unfallchirurgie

Hand- und Wiederherstellungschirurgie Steinhövelstr. 9

D-89075 Ulm 